\title{
A bilateral pneumonia following Thai herbal inhaler compulsive use
}

\author{
Marco Confalonieri ${ }^{1}$ \\ Mario Santagiuliana ${ }^{1}$ \\ Giovanni Falconieri² \\ Fulvia Martellani² \\ Elisa Baratella ${ }^{3}$ \\ Chiara Torregiani ${ }^{1}$
}

\author{
${ }^{1}$ Department of Pulmonology, University Hospital \\ of Cattinara, Trieste, Italy \\ 2 Institute of Pathology, University of Trieste, Trieste, \\ Italy \\ ${ }^{3}$ Department of Radiology, University Hospital \\ of Trieste, Trieste, Italy
}

\author{
Address for correspondence: \\ Marco Confalonieri \\ Department of Pulmonology \\ University Hospital of Cattinara \\ Trieste, Italy \\ E-mail: marco.confalonieri@aots.sanita.fvg.it
}

\section{Summary}

Traditional herbal therapy is often underestimated as a possible cause of drug-induced lung diseases. We report the case of a 51 years old man who developed acute respiratory failure after abuse and misuse of a traditional Thai herbal inhaler. The composition of the herbal therapy was only partially known. However, we supposed that the menthol balm may have been responsible of the pulmonary lipoid inclusions and trans-anethole may have caused diffuse alveolar lung damage.

KEY WORDS: diffuse parenchymal lung disease, herbal therapy, drug-induced lung disease, ARDS.

A 51 years old man was admitted to the Cardiology Unit because of sudden chest pain, palpitations and shortness of breath. He was a worker in a manufacturing company without any particular dust or metal exposition. Moreover, he was an occasional drummer and a mild smoker, without a relevant past history. Until those sudden symptoms, he didn't have previously exercise limitation. At hospital cardiologists promptly diagnosed the rupture of a tendon cord of the mitral valve with severe mitral failure, and planned cardiosurgery. During this first hospitalization, the patient had some episodes of paroxysmal supra-ventricular tachycardia (max. 135 bpm), then solved by adenosine infusion.

The patient developed a small "hospital-acquired pneumonia" at the right lower lobe, so an antibiotic therapy (levofloxacin 500mg os b.i.d.) was added at hospital discharge.

After seven days from discharge, the patient was another time admitted to the hospital because of progressive dyspnea and hemoptysis. He had cyanosis, tachypnea, tachycardia, mild fever, but no apparent signs of heart failure. Radiology imaging (chest X-ray and thorax CT scan) showed bilateral shadows consistent with diffuse pneumonia (Figure 1). Other main findings at the second admission were: hypoxemia and hypocapnia $\left(\mathrm{PaO} 250 \mathrm{mmHg}, \mathrm{PaCO}_{2} 33 \mathrm{mmHg}\right.$ ), elevated reactive C-protein (163 $\mathrm{mg} / \mathrm{L}))$, increased blood eosinophils (9.8\%), normal D-dimer and BNP, negative autoimmunity.

A bronchoscopy was then performed to obtain bronchoalveolar lavage (BAL) and lung transbronchial cryobpsies. The BAL resulted not diagnostically significant, nor an infectious agent was identified. The cryobpsy showed a picture typical of an inhalation pneumonia with lipid intracellular droplets in some alveolar macrophage, but without be really diagnostic for lipoid pneumonia (Figure 2). The pathologists observed also patchy organizing pneumonia and diffuse lung injury. So, the physicians questioned the patient again to realize which substances may have been inhaled quite recently, according to the quite rapid worsening of symptoms and development of bilateral pneumonia. After an accurate interrogation to the patient, we acknowledged that after the first episode of acute shortness of breath due to the rupture of mitralic tendon cord, the patient inhaled repeatedly a "natural" product given by a friend who was just come back from Thailand (Figure 3 ). The product was a small cylinder containing a lipid balm basis underlying a vegetal sac with dried herbs inside. The balm contained menthol and camphor, but the herbal content was not declared by the producer who stated that herbal mixture is a "secret receipt" indeed. On an external inspection basis the only herb easily recognizable was the star anise. The product is designed to be inhaled by the nostrils, but the patient continuous inhaled through the mouth keeping the product within his lips and inspiring deeply more and more that his breathless sensation worsened. So, we realized that a compulsive (ab)use of the inhaler for several days from the first hospital admission to the second one may have caused the inhalation pneumonia with associated acute alveolar injury. The patient was treated with corticosteroids (methylpred- 


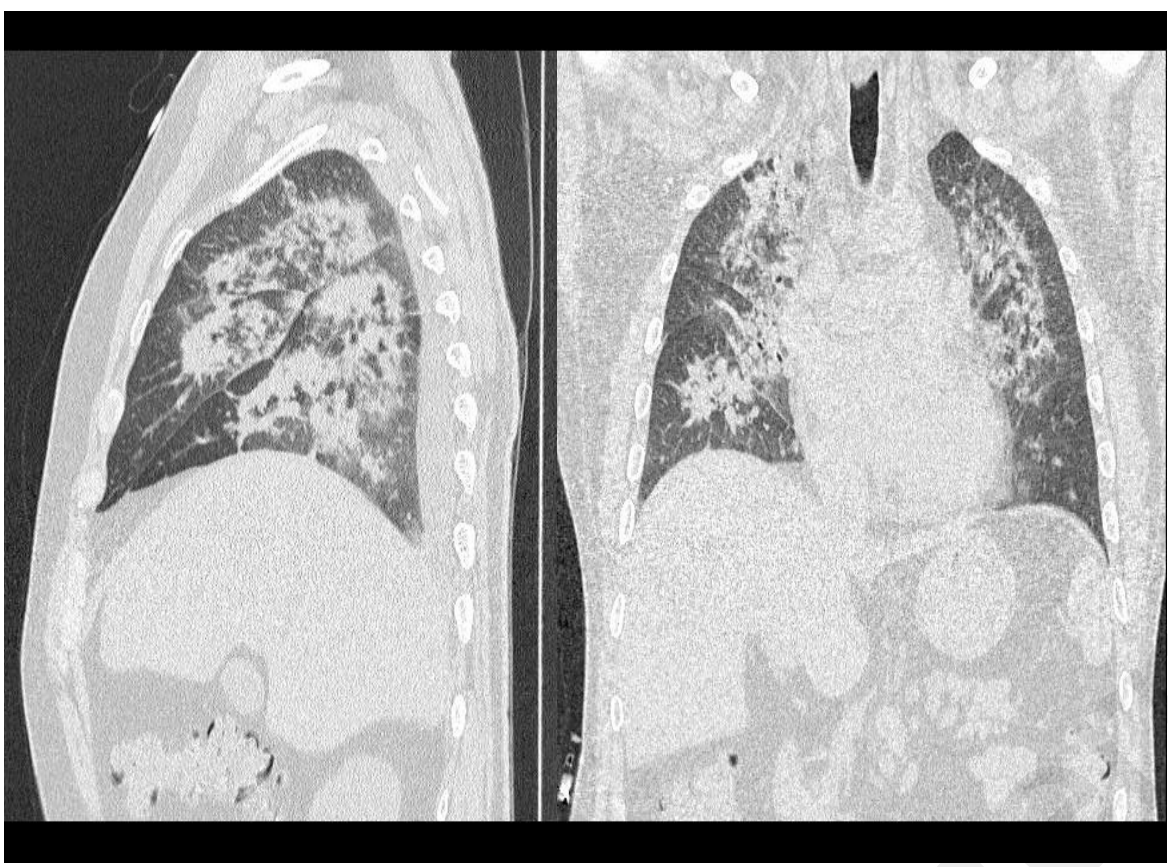

Figure 1 - Lateral and frontal images of thorax CT scan showing bilateral diffuse alveolar filling and interstitial thickening.

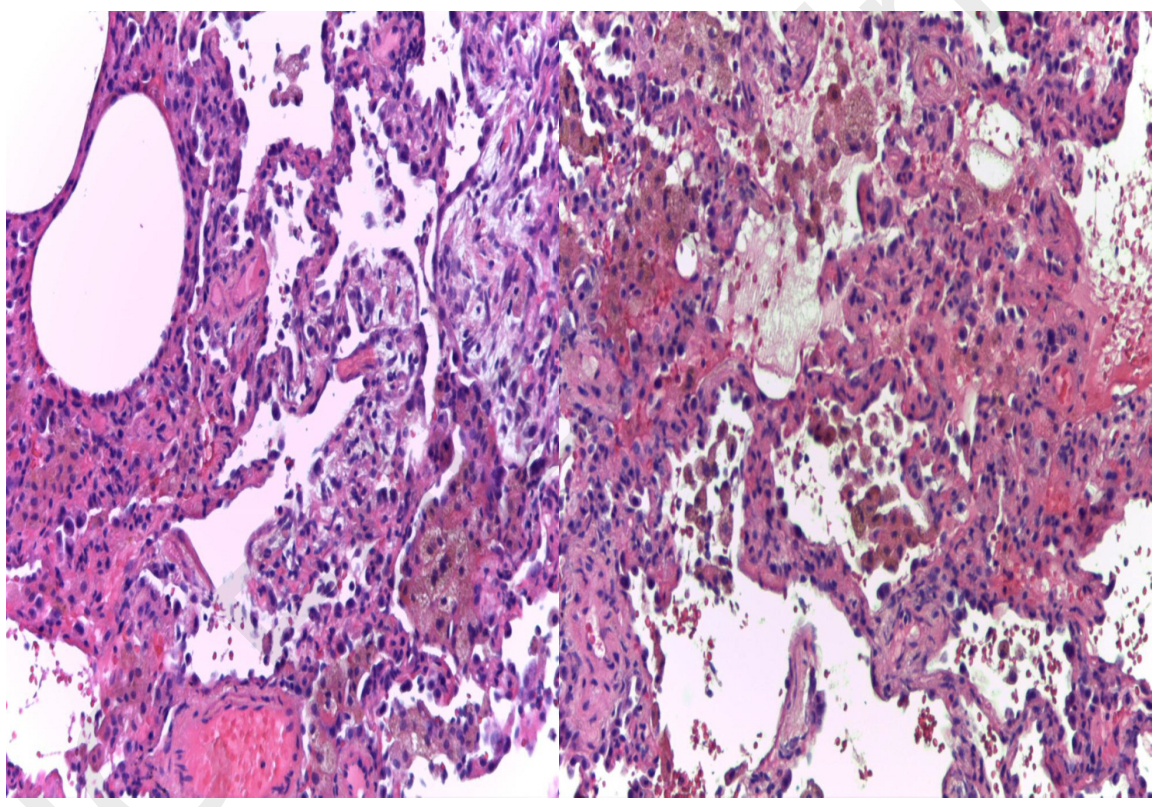

Figure 2 - Pathology of the lung lesions, obtained by bronchoscopic cryobiopsy.

nisolone $16 \mathrm{mg}$ t.i.d.) and cyclophosphamide tablets ( $2 \mathrm{mg} / \mathrm{kg}$ body weight/day) with quite rapid reduction of inflammation, improved gas exchange and symptoms, but most of radiological alterations persisted till more than weeks before to disappear.

\section{Discussion}

Inhalation pneumonia due to exogenous lipoids and chemical compounds is an uncommon condition that should not be confounded with aspiration pneumonitis or Mendelson's syndrome due to aspiration of gastric acid (1). Chest radiology imaging in patients with chemical pneumonia is characterized by the presence of infiltrates, predominantly the alveolar type pattern, sometimes simulating the appearance of pulmonary edema. Herbal drugs may cause various types of pneumotoxicity, from acute/subacute pneumonitis to chronic fibrosing diffuse lung disease $(2,3)$. Also, balm inhalation has been reported to cause lipoid accumulation in the lungs in some circumstances $(4,5)$. 


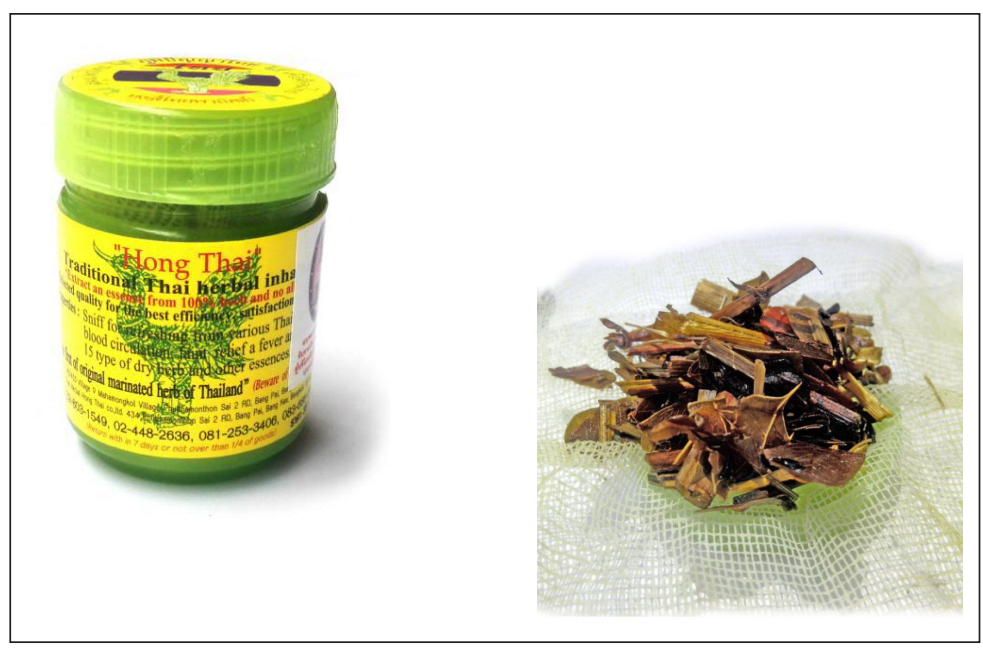

Figure 3 - The Hong Thai traditional herbal inhaler.

The present case suggests a temporal relationship between the development of lung injury and the prolonged incorrect inhalation of a "natural" product of vegetal origin. The pathology of the lung showed the presence of either exogenous lipoid material inside the alveolar macrophages and lung injury characterized by interstitial inflammation, organizing pneumonia, and alveolar damage. We are aware that the patient may have introduced lipoid droplets into his lungs with repetitive deep inhalations throughout the mouth, but this case show also a possible lung damage due to the herbal components of a diffuse uncontrolled product available on the web for healthcare purpose (6). The present case fulfills at least three out of the five diagnostic criteria for drug-induced infiltrative lung disease according to Camus et al. (7). Unfortunately, we were not able to identify completely the composition of the herbal mix contained in the traditional Hong Thai inhaler that the patient continuously aspirated by his mouth for several days. Nevertheless, a single herb surely identifiable in that product was the star anise, well recognizable thanks to its singular star shape. Chinese star anise or Illicium verum, is a quite common evergreen tree of Southwest Asia often used for traditional Chinese and Thai medicine preparations (8). The Japanese star anise or Illicium anisatum is a poisonous tree with toxic effects. It is impossible to distinguish Chinese star anise from Japanese star anise in its appearance only, due to morphological similarities between the species. Nevertheless, the Chinese star anise has been reported to be toxic in some pediatric cases (9), and its main component trans-anethole may be a chemical precursor to paramethoxyamphetamine (PMA), an illicit drug with neurological and systemic noxious effects (10). Diluiting anethole with water-based liquid a spontaneous microemulsion, called "ouzo effect" which may have acted as a carrier for other substance and a promoter of the lung damage in the present case (11). Dedicated investigations on the safety of anethole for inhalation does not exist. However, the lung damage occurred in the present case may be due to this, but also to any other component of the "secret" herbal mix. In fact, instructions on the Hong Thai inhaler's box suggest that the product should be used to sniff for the relief of superior airways disturbances, but no alert exists about possible damage from its bad use nor the exact herbal composition is declared. In conclusion, the present case may give a warning on the misuse of self-declared safe herbal compound for inhalation use.

\section{References}

1. Marik PE. Aspiration pneumonitis and aspiration pneumonia. N Engl J Med. 2001 Mar 1;344(9):66571.

2. Camus P, Foucher P. The drug-induced respiratory disease website. www.pneumotox.com/drug/view/ 569/herbals-herbal-therapy-plants/

3. Inamura T, Oymada Y, Asano K, Yamaguchi K. Pneumonitis induced by Ou-gon (Scullcap). Intern Med. 2001;40:764-8.

4. Kulkarni T, Pena T. A case of mistaken identity: lipoid pneumonia presenting as spiculated nodules after prolonged use of Vicks-vaporub. Am J Respir Crit Care Med. 2012;185:A6906.

5. Betancourt SL, Martinez-Guimenez S, Rossi SE, et al. Lipoid pneumonia: spectrum of clinical and radiologic manifestations. AJR. 2010;194:103-9.

6. http://www.amazon.com/Hong-thai-Traditionalherbal-inhalant/dp/B009FH0CWU.

7. Camus P, Fanton A, Bonniaud P, et al. Interstitial lung disease induced by drugs and radiation. Respiration. 2004;71:301-326.

8. Wang GW, Hu WT, Huang BK, Qin LP. Illicium verum: A review on its botany, traditional use, chemistry and pharmacology. Journal of Ethnopharmacology. 2011;136(1):10-20.

9. Perret C, Tabin R, Marcoz JP, et al. Apparent lifethreatening event in infants: Think about star anise intoxication! Archives de Pediatrie. 2011;18:750-3. 
10. Waumans D, Hermans B, Bruneel N, Tytgat J. A neolignan type impurity arising from the peracid oxidation reaction of anethole in the surreptitious synthesis of 4-methoxyamphetamine (PMA). Forensic
Science International. 2004;143:133-9.

11. Spernath A, Aserin A. Microemulsions as carriers for drugs and nutraceuticals. Adv Colloid Interface Sci. 2006;128-130:47-64. 